\title{
DNA methylation in repeat negative prostate biopsies as a marker of missed prostate cancer
}

\author{
Valentina Fiano ${ }^{1 *}$ (D), Daniela Zugna', Chiara Grasso', Morena Trevisan', Luisa Delsedime², Luca Molinaro², \\ Paola Cassoni ${ }^{3}$, Mauro Papotti ${ }^{4}$, Franco Merletti ${ }^{1,5}$, Olof Akre ${ }^{6}$, Andreas Pettersson ${ }^{7}$, Laura De Marco ${ }^{1,5}$ and \\ Lorenzo Richiardi ${ }^{1,5}$
}

\begin{abstract}
Background: Men often undergo repeat prostate biopsies because of suspicion of missed cancer. We assessed if (i) methylation of selected genes in prostate tissue vary with aging and (ii) methylation alterations in repeat biopsies predict missed prostate cancer.

Methods: We conducted a case-control study among men who underwent at least two negative prostate biopsies followed by a sampling either positive (cases $n=111$ ) or negative (controls $n=129$ ) for prostate cancer between 1995 and 2014 at the University Hospital (Turin, Italy). Two pathology wards were included for replication purposes. We analyzed methylation of GSTP1, APC, PITX2, C1orf114, GABRE, and LINE-1 in the first two negative biopsies. Conditional logistic regression was used to estimate odds ratios (ORs) and $95 \%$ confidence intervals (Cls) of the association between genes methylation and prostate cancer.

Results: Age at biopsy and time interval between the two negative biopsies were not associated with methylation levels of the selected genes in neither cases nor controls. GSTP1 methylation in the first and in the second negative biopsy was associated with prostate cancer detection [OR per 1\% increase: 1.14 (95\% Cl 1.01-1.29) for the second biopsy and 1.21 (95\% Cl 1.07-1.37) for the highest methylation level (first or second biopsy)]. A threshold $>10 \%$ for GSTP1 methylation corresponded to a specificity of 0.98 (positive likelihood ratio 7.87). No clear association was found for the other genes. Results were consistent between wards.
\end{abstract}

Conclusions: Our results suggest that GSTP1 methylation in negative prostate biopsies is stable over time and can predict missed cancer with high specificity.

Keywords: DNA methylation, Negative prostate biopsies, Prostate cancer, Prostate cancer diagnosis

\section{Background}

Men with elevated prostate-specific antigen (PSA) levels and/or a digital rectal examination suspicious of cancer usually undergo standard prostate biopsy to confirm the presence of cancer [1,2]. Prostate biopsies are affected by sampling error and have a false negative rate from $10 \%$ up to $30 \%$ [3-6]. As a consequence, many patients with a negative biopsy undergo one or several repeat biopsies, which are associated with pain, bleeding, and chance of

\footnotetext{
*Correspondence: valentina.fiano@unito.it

${ }^{1}$ Cancer Epidemiology Unit-CeRMS, Department of Medical Sciences, University of Turin and CPO Piemonte, Via Santena 7, 10126 Turin, Italy Full list of author information is available at the end of the article
}

serious infections from 0 to $6.3 \%[7,8]$. Although multiparametric magnetic resonance imaging (mp-MRI)-guided biopsies reduce the problem of false negative biopsies, the suspicion of cancer can remain high in a man with a previous negative round of biopsies [2].

Hence, there is a clinical need to identify diagnostic markers in morphologically benign tissue that can reduce the rate of repeat biopsies and missed cancer.

Several studies have shown that DNA hypermethylation of selected genes is present in non-tumor prostate tissue of men with prostate cancer, suggesting a diagnostic potential of aberrant DNA methylation in non-tumor tissue [9-15]. The biology of these alterations, if they

(C) The Author(s). 2019 Open Access This article is distributed under the terms of the Creative Commons Attribution 4.0 International License (http://creativecommons.org/licenses/by/4.0/), which permits unrestricted use, distribution, and 
change over time, and their potential clinical value are however poorly understood.

We studied men who underwent repeat prostate biopsies to understand (i) whether methylation alterations of selected genes in negative prostate tissue change over time and with aging or depend only on the specific characteristics of the prostate tissue in the sampled location and (ii) whether information on methylation alterations in repeat negative biopsies can be combined to predict the probability of a missed prostate cancer. We analyzed methylation of two genes [GSTP1 (glutathione S-transferase P1) and APC (adenomatous polyposis coli)] suggested as potential diagnostic markers for prostate cancer [12-15], of three genes [C1orf114 (chromosome 1 open reading frame 114), GABRE (gamma-aminobutyric acid receptor subunit epsilon), PITX2 (paired-like homeodomain transcription factor 2)] previously associated with prostate cancer prognosis [16-18], and of LINE-1 (long interspersed element-1), a marker of global methylation and a potential diagnostic and prognostic marker for prostate cancer [15-19].

\section{Results}

The study population of this case-control study involved two wards and was nested among 18,402 patients who underwent at least one prostate sampling [i.e., biopsy, transurethral resection of prostate (TURP), or partial prostatectomy] between 1995 and 2014 at the Italian University Hospital "Città della Salute e della Scienza di Torino", Torino, Italy. Cases and controls were defined as patients who underwent at least two biopsies negative for prostate cancer followed by a final sampling (i.e., the index sampling) that was either positive (cases) or negative (controls) for prostate cancer (Additional file 1: Figure S1). The study included 111 cases (86 Ward I and 25 Ward II) and 129 controls (100 Ward I and 29 Ward II); 34 subjects overlapped with a previous study [15]. Characteristics, including methylation levels, of cases and controls are reported in Table 1.

Additional file 1: Figure S2 reports the pairwise correlations between methylation levels of each selected gene within the first negative biopsy of cases and controls. All correlations were positive both in cases and controls, with an average correlation of 0.22 in cases and 0.20 in controls. The correlations were in general lower for LINE-1 than for the five selected genes, for which the highest estimates were observed for APC and PITX2 among cases $(r=0.48, p$ value $=<0.001)$ and $A P C$ and GSTP1 among controls $(r=0.39, p$ value $=<0.001)$. Similar results (not shown) were found when the analyses were carried out in the second biopsy. With the exception of the correlation between methylation levels in PITX2 and GSTP1 and PITX2 and APC ( $p=0.008$ and $p=0.017$ respectively), there was no evidence of difference between cases and controls in the within biopsy gene-specific correlation coefficients (all $p$ values $>0.10$ ).

As shown in Additional file 2: Table S1, there was no evidence of gene-specific pairwise correlation between the first and the second biopsy, which were not necessarily matched on the same prostate anatomical region, with the exception of $L I N E-1$, for which we estimated an $r$ of $0.34(p<0.0001)$ in cases and $0.28(p=0.001)$ in controls. A positive correlation was also observed for GSTP1 among controls $(r=0.23, p=0.02)$, but less so among cases $(r=0.12, p=0.25)$.

\section{Methylation changes in association with age and time between biopsies in cases and controls}

We evaluated the change in methylation levels with time in the prostate tissue of cases and controls using, first, a cross-sectional approach in which we assessed the association between age at the first biopsy and methylation levels of the selected genes, and second, a longitudinal approach in which we assessed the association between, on the one hand, the time between the first and the second biopsy and, on the other hand, the difference in methylation of each selected genes between the second and the first biopsy. The first, cross-sectional approach is potentially biased by patients' heterogeneities with age at diagnosis, while the second, longitudinal approach is conducted within patients, and thus is not affected by their heterogeneities. Both in cases and controls, age at first biopsy was not associated with methylation levels in any of the selected genes; although there was variability in methylation levels among both cases and controls, the median levels remained constant with age (Fig. 1). The results were similar when we analyzed the association between age and methylation levels in the second biopsy (data not shown). The predicted values of gene-specific median methylation levels at the first biopsy at selected ages $(55,60,65$, 70 , and 75 years) with $95 \%$ confidence intervals are reported in Additional file 2: Table S2.

Consistently, analyses on the difference in methylation levels between the two biopsies of each selected gene by time interval revealed that, although there was a large variability in the differences of methylation levels between the first and second biopsies both among cases and controls, the median difference did not change over time for any of the genes (Fig. 2). The predicted values of the median differences in gene-specific methylation levels between the two biopsies, at selected time intervals $(10,20,40,60,80$, and 100 months), with $95 \%$ confidence intervals, are reported in Additional file 2: Table S3. The estimates were close to the null value and there was no evidence of a deviation from the null value over time. 
Table 1 Characteristics of cases and controls

\begin{tabular}{|c|c|c|}
\hline Characteristic & Cases $(n=111)$ & Controls $(n=129)$ \\
\hline Age at first biopsy, median years (IQR) (missing: 0) & $66.2(61.9-70.7)$ & $65.6(59.5-69.2)$ \\
\hline Calendar year at first biopsy, median (IQR) (missing: 0) & $2002(98-04)$ & $2003(01-05)$ \\
\hline Time interval between the first and second biopsy, median months (IQR) (missing: 0) & $21.9(11.9-37.1)$ & $22.5(12.7-41.5)$ \\
\hline Time interval between the first biopsy and the index sampling, median months (IQR) (missing: 0) & $44.5(27.2-74.3)$ & $45.9(27.1-73.5)$ \\
\hline \multicolumn{3}{|l|}{ Number of patients per ward (\%) (missing: 0) } \\
\hline Ward I & $86(77.5)$ & $100(77.5)$ \\
\hline Ward II & $25(22.5)$ & $29(22.5)$ \\
\hline \multicolumn{3}{|l|}{ GSTP1 methylation \%, median (IQR) } \\
\hline First biopsy (missing: 8 cases, 14 controls) & $3.25(1.75-5.50)$ & $2.75(1.37-4.25)$ \\
\hline Second biopsy (missing: 7 cases, 8 controls) & $3.25(2.00-6.00)$ & $2.75(1.75-3.75)$ \\
\hline Difference between the second and the first biopsy (missing: 14 cases, 20 controls) & $0.00(-2.25-2.25)$ & $0.00(-1.00-1.25)$ \\
\hline \multicolumn{3}{|l|}{ PITX2 methylation \%, median (IQR) } \\
\hline First biopsy (missing: 18 cases, 23 controls) & $8.75(4.75-11.75)$ & $8.25(5.12-11.19)$ \\
\hline Second biopsy (missing: 17 cases, 14 controls) & $8.25(5.50-12.19)$ & $9.00(5.75-12.87)$ \\
\hline Difference between the second and the first biopsy (missing: 30 cases, 30 controls) & $-1.00(-6.00-5.25)$ & $0.00(-3.62-4.25)$ \\
\hline \multicolumn{3}{|l|}{ APC methylation \%, median (IQR) } \\
\hline First biopsy (missing: 15 cases, 21 controls) & $1.33(1.00-3.08)$ & $1.33(1.33,3.33)$ \\
\hline Second biopsy (missing: 16 cases, 17 controls) & $1.33(1.00-3.92)$ & $1.33(1.00-3.67)$ \\
\hline Difference between the second and the first biopsy (missing: 29 cases, 33 controls) & $0.00(-1.33-1.83)$ & $0.17(-0.33-1.42)$ \\
\hline \multicolumn{3}{|l|}{ Clorf114 methylation \%, median (IQR) } \\
\hline First biopsy (missing: 18 cases, 19 controls) & $4.00(2.00-8.50)$ & $2.50(1.50-5.50)$ \\
\hline Second biopsy (missing: 12 cases, 9 controls) & $5.00(1.50-9.25)$ & $4.50(1.50-7.12)$ \\
\hline Difference between the second and the first biopsy (missing: 28 cases, 23 controls) & $0.00(-3.25-4.50)$ & $0.50(-2.00-4.50)$ \\
\hline \multicolumn{3}{|l|}{ GABRE methylation \%, median (IQR) } \\
\hline First biopsy (missing: 27 cases, 38 controls) & $2.70(1.00-6.30)$ & $2.00(1.00-4.90)$ \\
\hline Second biopsy (missing: 28 cases, 25 controls) & $2.60(1.00-5.50)$ & $1.90(1.00-5.20)$ \\
\hline Difference between the second and the first biopsy (missing: 44 cases, 51 controls) & $-0.20(-1.90-2.40)$ & $0.00(-2.00-1.80)$ \\
\hline \multicolumn{3}{|l|}{ LINE-1 methylation \%, median (IQR) } \\
\hline First biopsy (missing: 5 cases, 7 controls) & $69.7(66.0-74.0)$ & $69.3(66.3-72.6)$ \\
\hline Second biopsy (missing: 3 cases, 3 controls) & $69.3(66.2-72.1)$ & $69.0(66.1-73.0)$ \\
\hline Difference between the second and the first biopsy (missing: 24 cases, 30 controls) & $0.00(-3.33-4.00)$ & $0.17(-4.00-4.08)$ \\
\hline \multicolumn{3}{|l|}{ Gleason score (missing: 2 cases) } \\
\hline 6 & $28(25.7)$ & - \\
\hline $3+4$ & $53(48.6)$ & - \\
\hline $4+3$ & $13(11.9)$ & - \\
\hline $8+$ & $15(13.8)$ & - \\
\hline PSA at the second biopsy, median (IQR) (missing: 22 cases, 19 controls) & $9.15(6.02-13.00)$ & $8.00(6.00-10.16)$ \\
\hline
\end{tabular}

IQR interquartile range, $P S A$ prostatic-specific antigen

\section{Methylation levels in selected genes and risk of prostate cancer}

We estimated the association between methylation levels of the selected genes and the risk of prostate cancer detection (Table 2). The methylation level was treated as a continuous variable, and results were reported as the odds ratio (OR) of prostate cancer detection for a $1 \%$ increase in methylation. The main analyses focused on the methylation levels in the second biopsy in association with the risk of prostate cancer detection in the third sampling. We focused on the methylation levels in the second negative biopsy, instead of the first biopsy, as this study, by design, was constrained on having a second negative biopsy taken after the initial first negative 


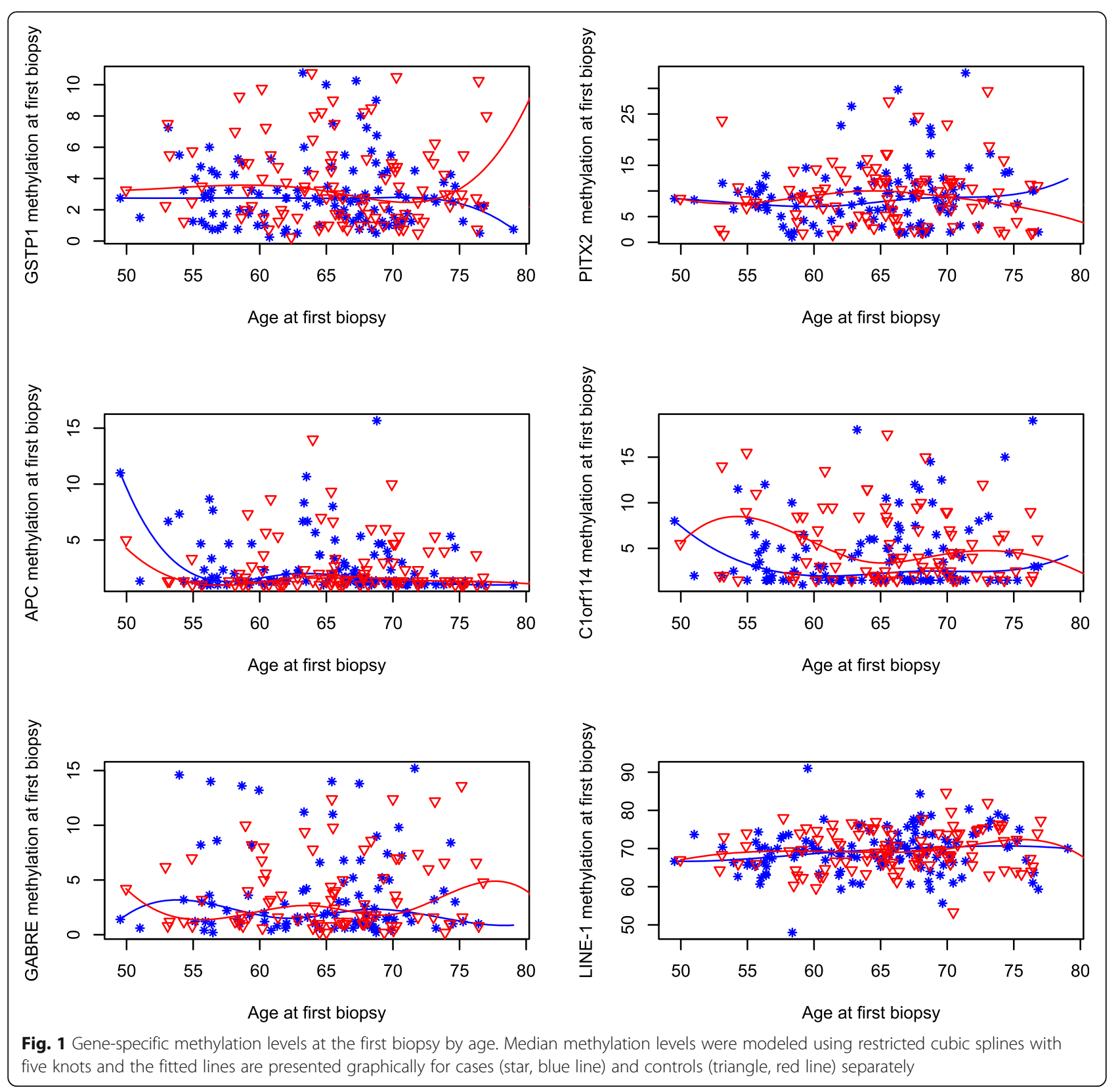

biopsy (see the "Methods" section for further details). We also analyzed the highest methylation level (first or second biopsy) again in association with the risk of prostate cancer detection in the third sampling and for each of the selected genes. The methylation levels of GSTP1 in the negative biopsies were associated with the risk of cancer diagnosis in the last sampling: the OR per $1 \%$ increase in methylation level was 1.14 (95\% CI 1.01-1.29), and 1.21 (95\% CI 1.07-1.37), for the highest methylation level (Table 2). We found no association between methylation levels of PITX2, APC, GABRE, or LINE-1 and prostate cancer detection, but a weak association for C1orf114 (Table 2). When all five genes and LINE-1 were included in the same model, the association with GSTP1 changed only marginally (data not shown). The adjustment for PSA did not substantially change the estimates.

Limited to GSTP1, we also conducted analyses stratified by the Gleason score, to evaluate more aggressive (score of $4+3$, or at least 8 ) and less aggressive (score of 6 or $3+4$ ) prostate cancers, and by ward (Ward I and Ward II), for the purpose of validation. For GSTP1, the ORs for aggressive prostate cancer were similar or slightly higher compared to those for non-aggressive prostate cancer (Table 3). The association between GSTP1 methylation and prostate cancer was present in 


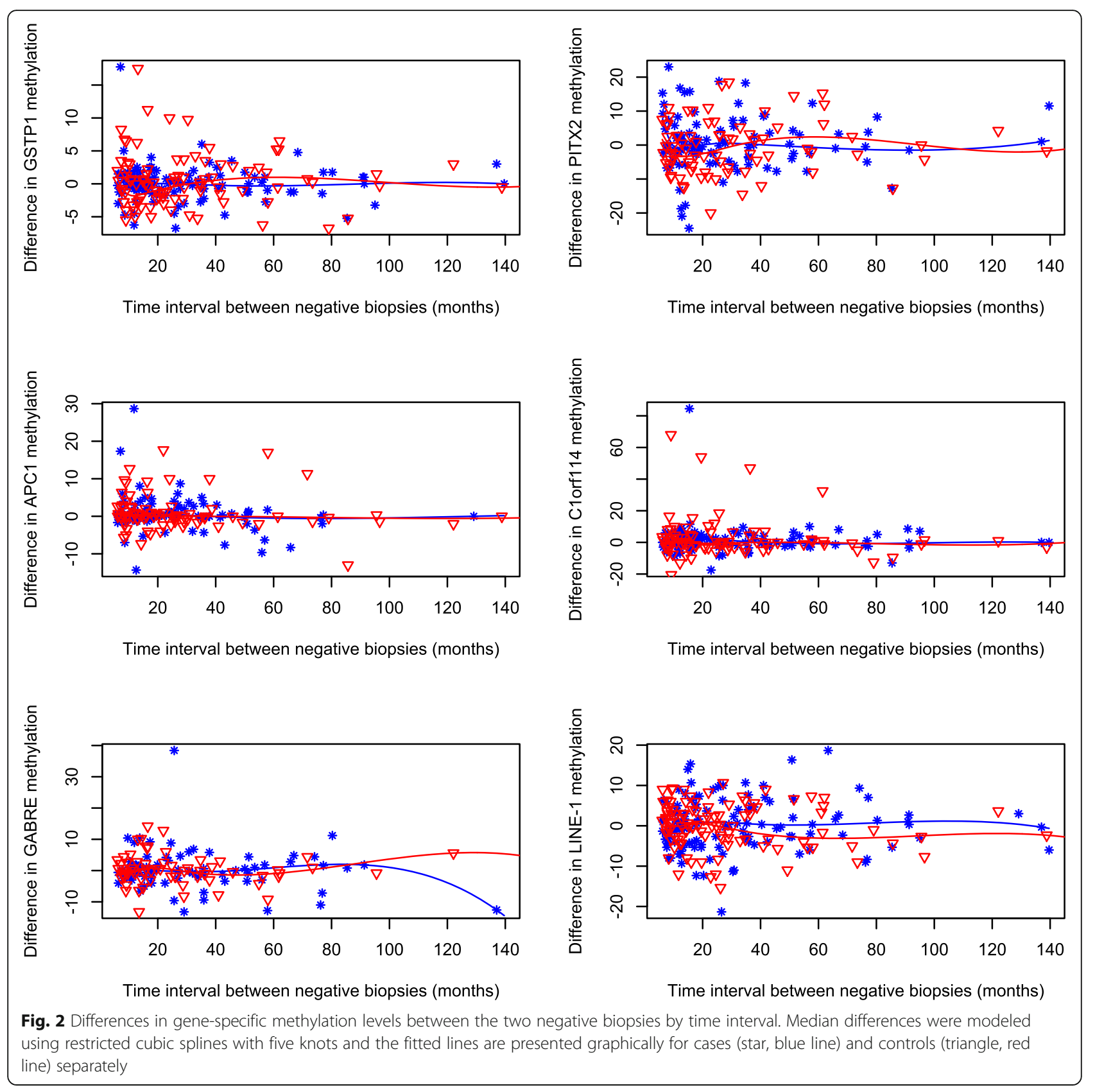

both Wards, even if confidence intervals were wide in Ward II due to a smaller sample size.

For GSTP1, a threshold $>10 \%$ revealed an OR of prostate cancer of 9.61 (95\% CI: 1.07-86.3) for methylation level, and of 5.10 (95\% CI: 1.33-19.6) for the highest methylation level. Table 4 reports the non-parametric values of specificity and sensitivity [and corresponding positive and negative likelihood ratios (LRs)] for different thresholds of GSTP1 methylation. The LRs, i.e., LR+ when the test is positive and LR- when the test is negative, are based on sensitivity and specificity and give a straightforward summary measure of the informative value of a test, as the post-test probability can be easily calculated as a function of the pre-test probability and the LR (post-test odds $=$ pre-test odds $\times$ LR).

A threshold of $>10 \%$ corresponded to a specificity of 0.98 and a LR+ of 7.87 , although sensitivity was low and LR- was close to 1.0 .

\section{Discussion}

We found, both in cases and controls, that neither the age at prostate biopsy nor the time interval between two negative biopsies were associated with methylation of GSTP1, APC, C1orf114, GABRE, PITX2, or LINE-1 in 
Table 2 Association between gene-specific methylation (considered as a continuous variable, per each 1\% increase) and the risk of prostate cancer detection

\begin{tabular}{|c|c|c|c|c|}
\hline \multirow[t]{2}{*}{ Gene } & \multicolumn{2}{|c|}{$\begin{array}{l}\text { ORs of prostate cancer for methylation level in the second } \\
\text { biopsy }\end{array}$} & \multicolumn{2}{|c|}{$\begin{array}{l}\text { ORs of prostate cancer for the highest }{ }^{\dagger} \text { methylation level between the first } \\
\text { and the second biopsy }\end{array}$} \\
\hline & OR1 (95\% Cl) & OR2 $(95 \% \mathrm{Cl})$ & OR1 $(95 \% \mathrm{Cl})$ & OR2 (95\% Cl) \\
\hline GSTP1 & 1.19 (1.06 to 1.33$)$ & 1.14 (1.01 to 1.29$)$ & 1.23 (1.10 to 1.39$)$ & 1.21 (1.07 to 1.37$)$ \\
\hline PITX2 & 0.98 (0.94 to 1.03$)$ & 0.99 (0.94 to 1.04$)$ & 1.02 (0.98 to 1.06$)$ & $1.02(0.97$ to 1.06$)$ \\
\hline$A P C$ & 1.02 (0.95 to 1.09$)$ & 1.01 (0.94 to 1.09 ) & 1.04 (0.97 to 1.10$)$ & 1.03 (0.96 to 1.10$)$ \\
\hline Clorf114 & 1.02 (0.99 to 1.06$)$ & 1.02 (0.98 to 1.05 ) & 1.04 (1.00 to 1.08$)$ & 1.03 (0.99 to 1.06$)$ \\
\hline GABRE & 1.00 (0.94 to 1.06$)$ & 0.99 (0.93 to 1.06$)$ & 1.00 (0.95 to 1.06$)$ & 1.00 (0.94 to 1.06$)$ \\
\hline LINE-1 & 1.01 (0.95 to 1.07 ) & 0.99 (0.92 to 1.06$)$ & 1.00 (0.93 to 1.07$)$ & 0.97 (0.90 to 1.04$)$ \\
\hline
\end{tabular}

OR odd ratio, $\mathrm{Cl}$ confidence interval

OR1 adjusted for the matching variables (ward and time distance between the first biopsy and the index sampling)

OR2 adjusted for the matching variables, age and year at the first biopsy and prostatic-specific antigen (PSA) at the second biopsy (continuous variables are centered at their mean)

${ }^{\dagger}$ for example: if methylation in GSTP1 is $4 \%$ in the first biopsy and 7\% in the second biopsy, the highest level used for this analysis is $7 \%$

non-tumor prostate tissue. This occurred even if there was a large variation in methylation levels both among patients and between two biopsies of the same patient, suggesting that methylation levels are specific to the specific sampling location in the prostate tissue, but do not follow specific changing patterns with time in histologically benign tissue. GSTP1 methylation in the first and the second negative biopsy was associated with the risk of cancer detection in the final sampling, while no clear association was found for the other genes. These results suggest that GSTP1 methylation in negative prostate biopsies is stable over time and that GSTP1 methylation can predict a missed cancer. Given the large differences in GSTPI methylation levels between patients' biopsies, these results suggest that the diagnostic value of GSTP1 methylation can be further improved by analyzing GSTP1 methylation levels in repeat biopsies.

A previous study by Kwabi-Addo and colleagues [20] on non-tumor prostate tissue, obtained from organ donors and patients who underwent cystoprostatectomy for bladder cancer, found that methylation of selected genes (including GSTP1) was positively associated with the patient's age. This finding is only in apparent contradiction with our results, as there are possible explanations. First, they included a large age range, from 17 to 84 years; from a visual inspection of Fig. 3 reported in the Kwabi-Addo and colleagues article [20], the slope of the association between methylation and age was strongly affected by patients aged less than 40 years, who were not included in our study as men are rarely scrutinized for prostate cancer at that age. The stability over time that we observed in our study may thus occur only at older ages. Second, the association between GSTP1 methylation and age was mainly due to seven cystoprostatectomy patients with much higher GSTP1 methylation levels than those found in non-tumor prostate tissues matched to prostate cancer tissues of 12 patients included in the study. In our study, the lack of association

Table 3 Association between GSTP1 methylation (considered as a continuous variable, per each 1\% increase) and the risk of prostate cancer detection stratified by Gleason score and ward

\begin{tabular}{|c|c|c|c|c|}
\hline \multirow[t]{2}{*}{ GSTP1 } & \multicolumn{2}{|c|}{$\begin{array}{l}\text { ORs of prostate cancer for methylation level in } \\
\text { the second biopsy }\end{array}$} & \multicolumn{2}{|c|}{$\begin{array}{l}\text { ORs of prostate cancer for the highest }{ }^{\dagger} \text { methylation leve } \\
\text { between the first and second biopsy }\end{array}$} \\
\hline & OR1 (95\% Cl) & OR2 (95\% Cl) & OR1 $(95 \% \mathrm{Cl})$ & OR2 (95\% Cl) \\
\hline \multicolumn{5}{|l|}{ Gleason score } \\
\hline 6 or $3+4$ & $1.14(1.02$ to 1.27$)$ & $1.10(0.97$ to 1.25$)$ & 1.19 (1.06 to 1.35$)$ & 1.18 (1.03 to 1.34$)$ \\
\hline at least $4+3$ & $1.33(1.08$ to 1.62$)$ & $1.22(0.96$ to 1.57$)$ & $1.27(1.07$ to 1.51$)$ & 1.21 (0.98 to 1.48$)$ \\
\hline \multicolumn{5}{|l|}{ ward } \\
\hline Ward I (86 cases, 100 controls) & 1.17 (1.03 to 1.32$)$ & 1.13 (0.99 to 1.30$)$ & 1.19 (1.06 to 1.34$)$ & 1.17 (1.03 to 1.33$)$ \\
\hline Ward II (25 cases, 29 controls) & 1.26 (0.96 to 1.66$)$ & $1.08(0.77$ to 1.52$)$ & 1.61 (1.04 to 2.49$)$ & 1.58 (0.92 to 2.71$)$ \\
\hline
\end{tabular}

$O R$ odd ratio, $\mathrm{Cl}$ confidence interval

OR1 adjusted for matching variables (ward and time distance between the first biopsy and the index sampling)

OR2 adjusted for the matching variables, age and year at the first biopsy and prostatic specific antigen (PSA) at the second biopsy (continuous variables are centered at their mean)

${ }^{\dagger}$ For example, if methylation in GSTP1 is $4 \%$ in the first biopsy and $7 \%$ in the second biopsy, the highest level used for this analysis is $7 \%$ 
Table 4 Non-parametric estimates of sensitivity, specificity, positive and negative likelihood ratios of prostate cancer detection in the third sampling, for increasing thresholds (from > 5 to > 10\%) of GSTP1 methylation observed in the first and second negative biopsy; 97 cases and 109 controls with measured GSTP1 methylation in both the first and the second biopsy

\begin{tabular}{|c|c|c|c|c|c|c|c|c|}
\hline \multirow{2}{*}{$\begin{array}{l}\text { Methylation } \\
\text { threshold }\end{array}$} & $n$ positive cases & $n$ negative cases & $n$ positive controls & $n$ negative controls & Sens & Spec & LR+ & LR- \\
\hline & \multicolumn{8}{|c|}{ The highest methylation level between the first and second biopsy } \\
\hline$>5 \%$ & 44 & 53 & 22 & 87 & 0.45 & 0.80 & 2.25 & 0.68 \\
\hline$>6 \%$ & 32 & 65 & 12 & 97 & 0.33 & 0.89 & 3.00 & 0.75 \\
\hline$>7 \%$ & 27 & 70 & 8 & 101 & 0.28 & 0.93 & 3.79 & 0.78 \\
\hline$>8 \%$ & 19 & 78 & 4 & 105 & 0.20 & 0.96 & 5.34 & 0.83 \\
\hline$>9 \%$ & 17 & 80 & 3 & 106 & 0.17 & 0.97 & 6.37 & 0.85 \\
\hline$>10 \%$ & 14 & 83 & 2 & 107 & 0.14 & 0.98 & 7.87 & 0.87 \\
\hline
\end{tabular}

Sens sensitivity, Spec specificity, $L R$ likelihood ratio

between age at sampling and GSTP1 methylation was supported by results from the longitudinal analyses carried out within patients, which are not affected by patients' heterogeneity. Our results thus suggest that GSTP1 hypermethylation is not due to aging but rather probably due to epigenetic deregulations occurring early in cancerogenesis.

The finding of an association between GSTP1 hypermethylation in non-tumor prostate tissue from a negative biopsy and the risk of prostate cancer detection in a later biopsy was replicated in both wards included in our study. Our findings are also consistent with the results of previous studies on GSTP1 methylation in non-tumor tissue as a potential marker for prostate cancer $[9,10$, 13-15]. We found that it is possible to improve the prediction by combining information on GSTP1 hypermethylation from multiple negative biopsies, even when obtained some years apart. It is thus likely that the predictive information from different biopsy procedures resembles the same information that could be obtained by the analysis of multiple cores of the same biopsy procedure. Finally, we found that a high threshold of methylation of GSTP1 is associated with a very high specificity, suggesting that this test could identify missed prostate cancer with a minimal increase in false positives. GSTP1 methylation testing in non-tumor tissue could thus potentially be combined with tests with high sensitivity, such as targeted biopsy after mp-MRI $[2,6,21]$, to improve overall diagnostic accuracy.

Steward and colleagues [13] and others [9, 10] have suggested that the combination of methylation of $A P C$ and GSTP1 could outperform the use of methylation of GSTP1 alone. In our study, however, methylation of $A P C$ was not associated with prostate cancer detection. Interestingly, in the study by Steward and colleagues, GSTP1 methylation was associated with a LR+ of 3.1 and a LR- of 0.67; when GSTP1 was combined with $A P C$, the LR- improved to 0.53 , but the $\mathrm{LR}+$ worsen to 1.7 (calculated by us on the basis of the sensitivity and specificity estimates reported in their Table 2). These findings would suggest that, if the aim is to maximize specificity, GSTP1 testing alone could outperform the combination of $A P C$ and GSTP1. Depending on the possible clinical uses, it could be discussed on whether to maximize LR+ or LR- or both and whether adding $A P C$ methylation would actually improve the test or not. It should be also noted that in our study, we assessed different CpG sites and used a different technique to assess $A P C$ methylation than in the study by Stewart et al.

The fact that we did not find an association with prostate cancer detection for the other analyzed genes, with the possible exception of C1orf114, suggests that the association between GSTP1 methylation and prostate cancer is not a consequence of a general alteration of the DNA methylome; nevertheless, we found a non-negligible positive correlation between genes in the same biopsy, which is consistent with the concept that the methylation pattern is a local characteristic of the prostate tissue. The lack of association for the other genes may also imply that the alteration in their methylation is a later event in cancerogenesis. It should be noted that these findings are consistent with previous studies that linked methylation of those genes with prostate cancer progression [16-18].

Although to our knowledge this is the first study that analyzed the change in methylation between negative biopsies and its relationship with a missed prostate cancer, our study has limitations. First, our study was restricted to patients who received at least three biopsies and these results are not necessarily generalizable to patients at their first biopsy. Second, the sample size was not sufficient to obtain strong evidence on the potential specificity of the GSTP1 methylation for aggressive prostate cancer (as opposed to nonaggressive cancers). Third, we analyzed a limited number of CpGs for each selected gene; a larger number of CpGs could give a more precise estimate of the gene-specific methylation levels.

In conclusion, our data suggest that GSTP1 methylation in negative prostate biopsies is stable over time and can predict missed cancer with high specificity. 


\section{Methods}

\section{Study population}

We conducted a case-control study nested among 18, 402 patients who underwent at least one prostate sampling [i.e., biopsy, transurethral resection of prostate (TURP) or partial prostatectomy] between 1995 and 2014 at the Italian University Hospital "Città della Salute e della Scienza di Torino", Italy. For validation purposes, we involved both pathology wards at the hospital (hereafter identified as Ward I and Ward II). Out of the 18, 402 patients, we identified 761 patients who underwent at least two samplings negative for prostate cancer followed by a final sampling (i.e., the index sampling) that was either positive (potential cases, $N=230$ ) or negative for prostate cancer (non-cases, $N=531$ ) (Additional file 1: Figure S1). We excluded patients whose negative samplings obtained before the index sampling were taken less than 6 months apart. We also excluded patients whose at least one of the negative sampling was a TURP or a partial prostatectomy as the prostate transition zone has a different methylation profile than the prostate peripheral zone (52 cases and 95 non-cases) [15-19]. Then, we sampled controls and matched cases to controls with a 1:1 ratio, on the pathology ward and the time interval between the first biopsy and the index sampling. After visual inspection of the slides and reading of the pathology reports, we excluded 22 cases and 16 controls for whom at least one of the two negative samplings was not a biopsy, 1 control whose biopsy was obtained only from the transitional zone, 6 cases and 3 controls with inadequate amount of tissue for molecular analyses, 2 cases with prostate cancer in one of the first two biopsies, 4 cases with bladder cancer that were erroneously included in the initial series of patients and 6 cases who had a diagnoses of atypical small acinar proliferation (ASAP) or high-grade prostatic intraepithelial neoplasia (HGPIN) and not prostate cancer. Incomplete strata with no cases or no controls were excluded, leading to the exclusion of 7 cases and 1 control; 111 cases and 129 controls remained in the study (Additional file 1: Figure S1).

Information on age at biopsy, tumor histology, and age value was obtained from the pathology reports. Since information on PSA was available for at least $80 \%$ of the subjects at the second biopsy, and only for $59 \%$ of the cases and $67 \%$ of the controls at the first biopsy, we only kept information at the second biopsy in the study. For all subjects, we collected blocks of formalin-fixed paraffin-embedded (FFPE) prostate tissue from the first two negative biopsies. If more than one FFPE block was available, we randomly selected one block, which implies that the repeat samples analyzed for the same patient were not necessarily obtained from the same anatomical region. For cases and controls with more than two negative biopsies before the index sampling, we selected the first and the last available biopsy to maximize the time distance between the two negative biopsies.

The diagnostic slides of the cases were reviewed by an uropathologist to assign a standardized contemporary Gleason score. For two cases, the diagnostic slides could not be evaluated and the Gleason score was considered missing.

\section{Molecular analysis}

For each FFPE block of the two first negative biopsies, we cut 3 to 5 (10 $\mu \mathrm{m}$ thick) sequential sections and extracted DNA, avoiding areas of chronic inflammation and fibromuscular stroma, using the QIAamp ${ }^{\circ}$ DNA FFPE Tissue Kit (Qiagen, Hilden, Germany). Genomic DNA underwent bisulphite modification using the EpiTect 96 Bisulfite Kit (Qiagen) and methylation was analyzed using PyroMark Q24 MDx (Qiagen). PCR primers that amplify the gene promoter region containing the target $\mathrm{CpG}$ sites, and the sequencing primers were designed with the software PyroMark Assay Design 2.0 (Qiagen) (Additional file 2: Table S4). We performed PCR reactions using PyroMark PCR kit (Qiagen) following the manufacturer's instructions, except for the annealing temperature (Additional file 2: Table S4). Methylated and unmethylated controls (EpiTect Control DNA, methylated and EpiTect Control DNA, unmethylated, Qiagen) were included in each PCR and pyrosequencing run. For each gene, we calculated the mean methylation levels among the target $\mathrm{CpG}$ sites (3 for $A P C$ and LINE-1, 4 for GSTP1 and PITX2 and 5 for GABRE, 2 for C1orf114) as in general they were strongly correlated within the same gene.

For each matched case-control stratum, all DNA samples were analyzed in the same batch and randomly allocated within the plate. To preserve the matching within batches, we did not re-run analyses in case of failure, which ranged from 25 to $30 \%$ (GABRE) to approximately 5\% (GSTP1 and LINE-1).

\section{Statistical analysis}

We used the Spearman rank coefficient to estimate the pairwise correlation of methylation levels between genes in the same biopsy or between two patients' biopsies in the same gene. We applied the standard Fisher's $z$-transformation to the Spearman coefficients to compare between cases and controls the correlation estimates [22]. We then used the Benjamini-Yekuteli method to control for multiple comparisons [23].

Analyses of the change in methylation levels with time/aging were carried out in cases and controls separately. For each of the selected genes, we analyzed the association between (i) methylation levels and age at prostate biopsy; (ii) the difference in methylation levels 
between the first and the second biopsy and the time interval between the two biopsies. Both analyses provide information on whether time/aging is associated with increasing or decreasing methylation; the former through a cross-sectional observation of methylation levels in men aged 50 to 80 years (i.e., the age distribution among study subjects), and the latter through a within-subject longitudinal observation of changes in methylation levels on a time interval from 6 to 140 months (i.e., the minimum and maximum time interval between the first and the second negative biopsy). Because the distribution of the methylation levels did not meet the normality assumption, we used quantile regression to model the medians of the methylation levels and the difference in methylation levels between the first and the second sample [24]. Age and time were modeled using restricted cubic splines with five knots. Because of the difficulty in interpreting the coefficients for a variable modeled using splines, we calculated the predicted values (with 95\% confidence intervals) of gene-specific median methylation levels at the first biopsy at selected ages $(55,60,65$, 70, and 75 years) (Additional file 2: Table S2). Similarly, we calculated the predicted values of the median differences in gene-specific methylation levels between the two biopsies at selected time intervals $(10,20,40,60,80$, and 100 months) (Additional file 2: Table S3).

We imputed missing values in gene methylation using multiple imputation by chained equations (MICE), assuming that the data were missing at random (MAR) [25]. The imputation model included demographic and clinical characteristics, including PSA levels at the second biopsy, as well as the methylation levels of the selected genes. Gleason score was not imputed but it was used as a predictor in the imputation models. We created 20 imputed datasets and combined their estimates according to Rubin's rule.

For each imputed dataset, we used conditional logistic regression to estimate odds ratio (ORs) and corresponding 95\% confidence intervals (CIs) of the association between methylation levels and prostate cancer detection. Each of the genes was introduced separately in the model. All models were inherently adjusted for the matching variables (time distance between the two biopsies and ward) and batch. We further adjusted for age, calendar year at the first biopsy (both introduced as continuous and centered at their mean), and PSA levels at the second biopsy.

We investigated the association between methylation levels in the second biopsy and prostate cancer detection and, separately, the association between the highest methylation level (first or second biopsy) and prostate cancer detection. We did not investigate the association between methylation levels in the first biopsy and prostate cancer detection because the estimates would have been biased by the fact that, by design, we conditioned on the second sampling being negative for cancer.

Methylation was modeled as a continuous variable. For GSTP1, we also used a threshold of $>10 \%$, which was higher than the threshold of $\geq 5 \%$ that we used in a previous study [15], in order to enhance specificity. On the non-imputed data, we calculated non-parametric estimates of sensitivity, specificity, and positive and negative likelihood ratios (LRs) for levels of methylation from $>5$ to $>10 \%$.

The analyses described above were conducted on the whole study sample, separately by Ward I and Ward II for the purpose of validation, and separately for "aggressive" (Gleason score of at least $4+3$ ) and "non-aggressive" (Gleason score $<4+3$ ) prostate cancer.

\section{Supplementary information}

Supplementary information accompanies this paper at https://doi.org/10. 1186/s13148-019-0746-6.

Additional file 1: Figure S1. Flow-chart diagram showing the case and control selection. Figure S2. Spearman rank correlation coefficients between gene-specific methylation levels within biopsy in cases and controls.

Additional file 2: Table S1. Spearman correlation coefficients of genespecific methylation levels between the two biopsies in cases and controls. Table S2. Predicted values of gene-specific median methylation levels at the first biopsy at selected ages, using quantile regression with age modelled by restricted cubic splines. Table S3. Predicted values of the median differences in gene-specific methylation levels between the two biopsies, at selected time intervals, using quantile regression with time modelled by restricted cubic splines. Table S4. Gene IDs, primer sequences, pyrosequencing assays and PCR annealing temperatures and number of $\mathrm{CpG}$ analysed per gene.

\section{Abbreviations}

APC: Adenomatous polyposis coli; ASAP: Atypical small acinar proliferation; C1orf114: Chromosome 1 open reading frame 114; Cls: Confidence intervals; FFPE: Formalin fixed paraffin embedded; GABRE: Gamma-aminobutyric acid receptor subunit epsilon; GSTP1: Glutathione S-transferase P1; HGPIN: Highgrade prostatic intraepithelial neoplasia; LINE-1: Long interspersed element-1; LRs: Likelihood ratios; MAR: Missing at random; MICE: Multiple imputation by chained equations; mp-MRl: Multiparametric magnetic resonance imaging; ORs: Odds ratio; PITX2: Paired-like homeodomain transcription factor 2; PSA: Prostate-specific antigen; TURP: Transurethral resection of prostate

\section{Acknowledgements}

We acknowledge the support of the "Vale per la vita" Onlus Association that donated a thermal cycler instrument to our laboratory.

\section{Authors' contributions}

VF conceived and designed the study, collected and processed the samples, optimized and performed the experiments, contributed to the interpretation of analysis, and drafted the manuscript. DZ analyzed data and contributed to the interpretation of analysis. CG collected and processed the samples and optimized and performed the experiments. MT collected and processed the samples and optimized and performed the experiments. LD contributed to the collection and assembly of data. LM contributed to the collection and assembly of data. PC contributed to the collection and assembly of data and provided samples for the study. MP contributed to collection and assembly of data and provided samples for the study. FM contributed to the data interpretation. OA contributed to the data analysis and interpretation. AP conceived and designed the study. LDM contributed to collection and assembly of data. LR was the principal investigator of the study, obtained 
financial support, conceived and designed the study, contributed to the interpretation of analysis, and drafted the manuscript. All authors read and approved the final manuscript.

\section{Funding}

The study was supported by the Italian Association for Cancer Research (project IG 2015 Id.17723) and by the ex-60\% MIUR, and received funding specifically dedicated to the Department of Medical Sciences from Italian Ministry for Education, University and Research (MIUR) under the program "Dipartimenti di Eccellenza 2018 - 2022". Project n D15D18000410001.

\section{Availability of data and materials}

The datasets generated during and/or analyzed during the current study are available from the corresponding author on reasonable request of qualified researchers for the purpose of academic, non-commercial research.

\section{Ethics approval and consent to participate}

The study was approved by the Ethical Committee of the San Giovann Battista Hospital - CTO/CRF/Maria Adelaide Hospital of Turin; no specific consent was obtained from the study participants as the study is based on archived material at the pathology wards from 1995 onwards.

\section{Consent for publication}

Not applicable.

\section{Competing interests}

The authors declare that they have no competing interests.

\section{Author details}

'Cancer Epidemiology Unit-CeRMS, Department of Medical Sciences, University of Turin and CPO Piemonte, Via Santena 7, 10126 Turin, Italy. ${ }^{2}$ Pathology Unit, A.O.U. Città della Salute e della Scienza Hospital, Turin, Italy. ${ }^{3}$ Pathology Unit, Department of Medical Sciences, University of Turin, Turin, Italy. ${ }^{4}$ Pathology Unit, Department of Oncology, University of Turin, Turin, Italy. ${ }^{5}$ Cancer Epidemiology Unit, A.O.U. Città della Salute e della Scienza Hospital and CPO Piemonte, Turin, Italy. ${ }^{6}$ Department of Molecular Medicine and Surgery, Karolinska Institutet and Department of Urology, Karolinska University Hospital, SE-17176 Stockholm, Sweden. ${ }^{7}$ Clinical Epidemiology Unit, Department of Medicine, Solna, Karolinska Institutet, Stockholm, Sweden.

Received: 29 April 2019 Accepted: 22 September 2019

Published online: 30 October 2019

\section{References}

1. Mottet $\mathrm{N}$, et al. EAU-ESTRO-SIOG guidelines on prostate cancer. Part 1: screening, diagnosis, and local treatment with curative intent. Eur Urol. 2017:71(4):618-29.

2. Kasivisvanathan $V$, et al. MRI-targeted or standard biopsy for prostate-Cancer diagnosis. N Engl J Med. 2018:378(19):1767-77.

3. Bjurlin MA, Taneja SS. Standards for prostate biopsy. Curr Opin Urol. 2014; 24(2):155-61.

4. Siddiqui MM, et al. Comparison of MR/ultrasound fusion-guided biopsy with ultrasound-guided biopsy for the diagnosis of prostate cancer. JAMA. 2015; 313(4):390-7.

5. Klemann $\mathrm{N}$, et al. Risk of prostate cancer diagnosis and mortality in men with a benign initial transrectal ultrasound-guided biopsy set: a populationbased study. Lancet Oncol. 2017;18(2):221-9.

6. Ahmed HU, et al. Diagnostic accuracy of multi-parametric MRI and TRUS biopsy in prostate cancer (PROMIS): a paired validating confirmatory study. Lancet. 2017:389(10071):815-22.

7. Loeb S, et al. Systematic review of complications of prostate biopsy. Eur Urol. 2013;64(6):876-92.

8. Borghesi $\mathrm{M}$, et al. Complications after systematic, random, and imageguided prostate biopsy. Eur Urol. 2017;71(3):353-65.

9. Troyer DA, et al. Prostate cancer detected by methylated gene markers in histopathologically cancer-negative tissues from men with subsequent positive biopsies. Cancer Epidemiol Biomark Prev. 2009;18(10):2717-22.

10. Trock BJ, et al. Evaluation of GSTP1 and APC methylation as indicators for repeat biopsy in a high-risk cohort of men with negative initial prostate biopsies. BJU Int. 2011;110(1):56-62.
11. Truong $M$, et al. Using the epigenetic field defect to detect prostate cancer in biopsy negative patients. J Urol. 2013;189(6):2335-41.

12. Richiardi $L$, et al. Methylation of APC and GSTP1 in non-neoplastic tissue adjacent to prostate tumour and mortality from prostate cancer. PLoS One. 2013;8:e68162

13. Stewart GD, et al. Clinical utility of an epigenetic assay to detect occult prostate cancer in histopathologically negative biopsies: results of the MATLOC study. J Urol. 2013;189(3):1110-6.

14. Partin AW, et al. Clinical validation of an epigenetic assay to predict negative histopathological results in repeat prostate biopsies. J Urol. 2014; 192(4):1081-7.

15. Zelic R, et al. Global hypomethylation (LINE-1) and gene-specific hypermethylation (GSTP1) on initial negative prostate biopsy as markers of prostate cancer on a rebiopsy. Clin Cancer Res. 2016;22(4):984-92.

16. Haldrup $C_{\text {, }}$ et al. DNA methylation signatures for prediction of biochemical recurrence after radical prostatectomy of clinically localized prostate cancer. J Clin Oncol. 2013;31(26):3250-8.

17. Strand $\mathrm{SH}$, et al. Prognostic DNA methylation markers for prostate cancer. Int J Mol Sci. 2014;15(9):16544-76.

18. Blute ML Jr, et al. The epigenetics of prostate cancer diagnosis and prognosis: update on clinical applications. Curr Opin Urol. 2015;25(1):83-8.

19. Fiano $\mathrm{V}$, et al. LINE-1 methylation status in prostate cancer and nonneoplastic tissue adjacent to tumor in association with mortality. Epigenetics. 2017;12(1):11-8.

20. Kwabi-Addo B, et al. Age-related DNA methylation changes in normal human prostate tissues. Clin Cancer Res. 2007:13(13):3796-02.

21. Fütterer JJ, et al. Can clinically significant prostate cancer be detected with multiparametric magnetic resonance imaging? A systematic review of the literature. Eur Urol. 2015;68(6):1045-53.

22. Myers $L$ and Sirois MJ. Spearman correlation coefficients, differences between. In: Encyclopedia of Statistical Sciences. Wiley. 2006. doi: https:// doi.org/10.1002/0471667196.ess5050.pub2 Accessed 15 Aug 2006.

23. Benjamini $Y$, Yekutieli $D$. The control of the false discovery rate in multiple testing under dependency. Ann Stat. 2001;29(4):1165-88.

24. Koenker R. Quantile Regression. New York: Cambridge University Press; 2005

25. Van Buuren S, Groothuis-Oudshoon K. mice: Multivariate Imputation by Chained Equations in R. Journal of Statistical Software. 2011:45(3):1-67.

\section{Publisher's Note}

Springer Nature remains neutral with regard to jurisdictional claims in published maps and institutional affiliations.

Ready to submit your research? Choose BMC and benefit from:

- fast, convenient online submission

- thorough peer review by experienced researchers in your field

- rapid publication on acceptance

- support for research data, including large and complex data types

- gold Open Access which fosters wider collaboration and increased citations

- maximum visibility for your research: over $100 \mathrm{M}$ website views per year

At $\mathrm{BMC}$, research is always in progress.

Learn more biomedcentral.com/submissions 